\title{
Impact of Hub Location on the Performance of Wireless Body Area Networks for Fitness Applications
}

\author{
Vit Sipal \\ Technological University Dublin, vit.sipal@tudublin.ie \\ Domenico Gaetano \\ Technology, domenico.gaetano@mydit.ie \\ Patrick McEvoy \\ Technological University Dublin, patrick.mcevoy@tudublin.ie
}

See next page for additional authors

Follow this and additional works at: https://arrow.tudublin.ie/engscheleart2

Part of the Electrical and Electronics Commons, and the Electromagnetics and Photonics Commons

\section{Recommended Citation}

Sipal, V.; Gaetano, D.; McEvoy, P.; Ammann, M.J., "Impact of Hub Location on the Performance of Wireless Body Area Networks for Fitness Applications," Antennas and Wireless Propagation Letters, IEEE, vol.14, pp.1522,1525, 2015 DOI:10.1109/LAWP.2014.2376943

This Article is brought to you for free and open access by the School of Electrical and Electronic Engineering at ARROW@TU Dublin. It has been accepted for inclusion in Articles by an authorized administrator of ARROW@TU Dublin. For more information, please contact arrow.admin@tudublin.ie, aisling.coyne@tudublin.ie, gerard.connolly@tudublin.ie.

Funder: Science Foundation Ireland and the Irish Research Council 
Authors

Vit Sipal, Domenico Gaetano, Patrick McEvoy, and Max Ammann

This article is available at ARROW@TU Dublin: https://arrow.tudublin.ie/engscheleart2/92 


\title{
Impact of Hub Location on the Performance of Wireless Body Area Networks for Fitness Applications
}

\author{
Vit Sipal, Domenico Gaetano, Patrick McEvoy, Senior Member, IEEE \\ and Max J. Ammann, Senior Member, IEEE
}

\begin{abstract}
This letter compares the propagation properties of Wireless Body Area Networks for three different locations (head, foot and waist) of the hub/internet gateway on the human body. The wireless channels between the hubs and four nodes (chest, back, and upper arms) are measured for frequencies between 5 and $7 \mathrm{GHz}$ on a female and a male subject performing push-ups and squats. A framework using path gain and fade depth metrics in spider plots is used for cumulative performance description. The results show that the best overall performance is for a hub located on the temple and the worst overall performance is achieved for a hub on the waist. These results are expected to stimulate further research into the optimum hub placement on the human body.
\end{abstract}

Index Terms-WBAN, hub location, propagation

\section{INTRODUCTION}

$\mathrm{W}$ EARABLE ELECTRONICS is seen as the next major frontier for the electronics industry. Wireless body area networks (WBANs) will be crucial catalysts of this growth because they connect wearable devices into the Internet-ofThings and thus unlock the extra added value provided by the application layer [1].

In the next five years, the market for WBANs is expected to grow exponentially. For instance, in 2018, the demand for wireless chipsets for healthcare and fitness applications will reach 250 million units a year, which is more than a ten-times the figure from 2010 [1].

As a result, both academia and industry have intensified the research and development efforts focusing on WBANs. In terms of network architecture, the prevailing approach considers internet access via a two-hop network. The devices will connect via a short, low power on-body link with a hub/aggregator, which will facilitate the connection to the

Manuscript received August 24, 2014; revised October 01, 2014 and November 10, 2014; accepted November 25, 2014. Date of publication MMM DD, YYY; date of current version MMM DD, YYY. The work was supported by the Science Foundation Ireland grant 09/IN.1/I2652, and by the Government of Ireland Postdoctoral Fellowship in Engineering, Science and Technology from the Irish Research council.

V. Sipal, D. Gaetano, P. McEvoy and M.J. Ammann are with the Antenna $\&$ High Frequency Research Centre, Dublin Institute of Technology, Dublin 8, Ireland (e-mail: name.surname@dit.ie). internet using an off-body link [2]-[6].

Even though both on- and off-body channels have been studied in great detail for frequencies up to $10 \mathrm{GHz}$, the open literature almost unanimously assumes that the hub is located on the waist of the user. Exceptions are works [7]-[9], which discuss the benefits for a hub located on the footwear.

This letter studies the suitability of three hub locations in WBANs for fitness scenarios. Such an investigation is not completely new. For instance reference [10] studies cooperative communications in WBAN for three hub locations on the torso. However to our best knowledge, the open literature does not report any work focusing on a systematic selection of optimum hub location outside of the torso. Furthermore, this letter introduces the possibility of using spider plots, known e.g. from economy, as a mean for simple graphical performance comparison of WBANs with different hub locations.

The structure of the letter is as follows. First, the case for alternative 'non-waist' hub locations is presented. Secondly, the measured 'fitness scenario' is introduced and finally the results are discussed.

\section{Case for Alternative Hub Locations}

As mentioned in the introduction, almost all works studying the propagation in WBANs consider the hub/aggregator placed in the waist/hip area of the human body. This choice is made because it is assumed that the mobile phone will represent the hub/internet gateway, and phones are usually carried in this area. This is indeed the likely scenario, unless the integration of cellular technology into smart glasses [11] disrupts the ubiquitous dominance of mobile phones as the main gateway to mobile internet.

However, even though market studies predict an exponential growth in the WBANs, the reality usually lags behind the predictions. For instance, in 2000, a market study by Merrill Lynch predicted that the global annual production of Bluetooth chipsets would reach the 2 billion mark in 2005 [11]. The actual production in 2005 was 200 million units and the 2 billion mark was not reached until 2013 [1].

Therefore, it seems more realistic that the first proper WBANs with several nodes will appear in specialized fields such as the military, professional athletes, or law enforcement 
units [9]. For these applications, the hub position does not have to be on the waist/hip. Instead it can be used as an additional degree of freedom in the network design.

In fact, other locations offer additional benefits. For instance, in terms of propagation, it may be advantageous to locate the hub in the extremities of the body (head or foot) because the entire body can be covered by directional antennas [7]-[9]. Integration in the footwear enables extension of battery life by harvesting energy from piezoelectric elements integrated in the footwear [9]. Similarly, it may be advantageous to co-locate the hub and the video camera, located on the head, to spare the on-body link for the video feed which will require more data-rate than other sensors.

In order to aid these design decisions a measurement campaign and a framework providing a network performance comparison for different hub locations are introduced.

\section{MEASUREMENT CAMPAIGN}

Three WBAN architectures are compared: a waist-centric network (hub on the waist); a head-centric network (hub on the left temple) and a footwear centric network (hub on the left ankle). The hub locations are shown in Fig. 1.

For each hub position, four positions of WBAN nodes were compared. These positions, also introduced in Fig. 1, are: chest (sternum); back ( $4^{\text {th }}$ vertebrae); left and right upper arm.

The transfer functions of the wireless channel, $H(f)$, between the hubs and individual nodes were recorded using a Rohde \& Schwarz ZVB 8 vector network analyzer for two sets of exercises: push-up exercise and squat exercise. For each exercise and each node-hub combination 100 channel measurements were taken with a time step of $0.28 \mathrm{~s}$. During the measurement the subjects performed approx. 10 push-ups or 10 squats. The measurement was repeated for two test subjects: male $(175 \mathrm{~cm}, 80 \mathrm{~kg})$, and female $(160 \mathrm{~cm}, 57 \mathrm{~kg})$.

The push-up and squat exercises were selected to simulate a fitness scenario and compared to more traditional standing/walking scenarios [2]-[9], they result in a larger scale of relative limb motion with respect to the torso.

The measurement was performed in an indoor environment. In total 2001 data points were recorded for frequencies from 5 to $7 \mathrm{GHz}$. This band was selected because it comprises not only communication in the 5.2 and $5.8 \mathrm{GHz}$ ISM bands, but also in the 5-6 GHz band, which according to some reports might be approved for ISM use [12].

For the measurements two antenna types from Fig. 2 were used. For the nodes Vivaldi antennas were employed. The radiation was directed towards the hub for the case when the subject is standing, i.e. radiation towards the ground for waistand footwear-centric architecture and radiation towards the ceiling for the head-centric system.

The same Vivaldi antennas were used for the hubs in the

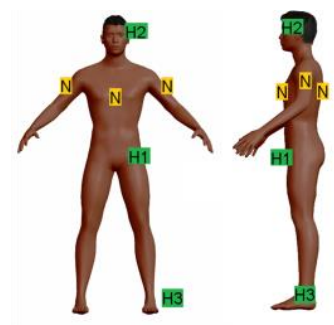

Fig. 1 Positions of hubs and nodes on the subject case of the head-centric (radiating to the ground) and footwear-centric (radiating to the ceiling) networks. For the waist-centric network, a spline monopole [8] had to be employed in order to comply with requirement to ensure

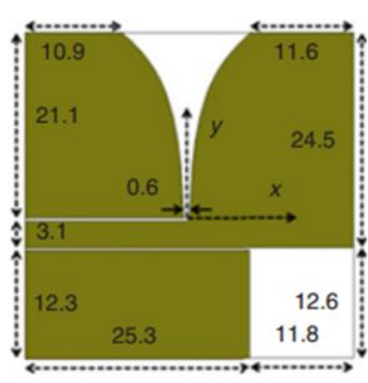

a)

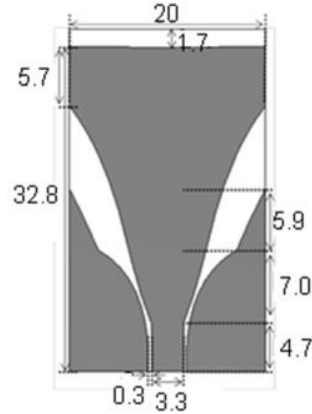

b)
Fig. 2 Antennas used for the experiment. Both antennas printed on flexible FR4 substrate with thickness $0.25 \mathrm{~mm}$ (a) Vivaldi antenna: curve eq. $x=$ $0.19 \exp (0.17 y)$ for $0<y<21 \mathrm{~mm}$ (b) spline monopole antenna.

Detailed description of the antennas and their performance is found in $[81$.

coverage for nodes that might be located both below and above the hub. The monopole's principle polarization was parallel with the ground for a standing subject.

To minimize the impact of electromagnetic (EM) waves creeping along the cables, ferrites were used at the ports of the antennas. Cable bends occurring during the exercise should not impact the channel transfer function because phase and amplitude stable cable were used. Body movement did result in changes in the orientation of the antennas. However, this would feature in normal WBAN use because of how sensors reorientate with body movement.

There is a certain systematic error in the measurement, but the errors are believed to correspond to the standard from other works [2]-[6]. Hence, the results are believed to provide a good qualitative description of the real scenarios.

\section{DATA ANALYSiS}

The measured data were analyzed for path-gain $P G$ measured over a bandwidth $B$ [9]:

$$
P G=10 \log \left\{\int_{f_{c}-B / 2}^{f_{c}+B / 2}|H(f)|^{2} d f\right\}
$$

where $H(f)$ is the measured transfer functions.

This letter considers $B=20 \mathrm{MHz}$, corresponding to the bandwidth used by the 802.11. In total 99 center frequencies $f_{c}$ were investigated (5.01-6.99 GHz spaced by $20 \mathrm{MHz}$ ).

A traditional approach to analysis of data is to plot the path gain as a function of time. This identifies the impact of body movements on the quality of the wireless link. An example path-gain for the squat exercise is shown in Fig. 3.

Another often used approach is to study the cumulative probability function (CPF). An example comparing path gain CPFs for a connection with a node on the back in three different network architectures is presented in Fig. 4. Using

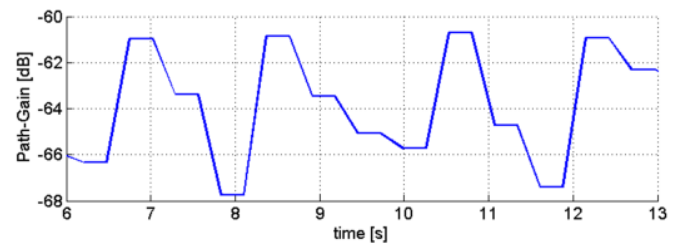

Fig. 3 Path-gain at the center frequency of $6 \mathrm{GHz}$ for a connection between the ankle and back during a squat exercise for female subject 


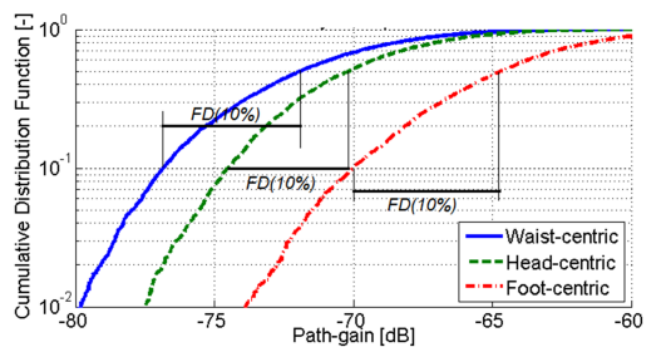

Fig. 4 Example cumulative probability function of path-gains for three hub locations for female subject node on the back.

CPFs allows an insight in the outage probabilities as well as the nature of the fading (Rician/Rayleigh).

Whilst the plots presented in Fig. 3 and 4 are suitable for a link analysis, they cannot be directly used to study the performance of the network architecture. This study measures the path gain between the hub and the node for 3 hub locations, 4 node locations, for 2 exercises and for 2 test subjects. That would result in $16 \mathrm{CPF}$ plots similar to Fig. 4.

Comparison of 16 graphs with three curves each does not allow for a simple assessment especially if neither hub location is expected to be significantly superior. Thus, the use of spider plots is proposed to facilitate the comparison.

Spider plots are often used in physics, economics or business where the performance of different systems needs to be compared in multiple criteria [14]. Each criterium represents a spoke of the net. A connection of the spokes then resembles a spider net. A good system is typically represented by a curve enclosing a large area whilst manifesting a good level of symmetry, i.e. good performance across all criteria.

Fig. 5 shows 6 spider plots which summarize the results of the measurements. The plots in the top half show the results of measurement on the female subject whereas the plots in the lower half are for the male subject. The upper half of each plot represents the push-up exercise whereas the lower half represents the squat exercise. Each half consists of four spokes representing the four node positions.

Fig. 5a (on the left) shows the median path-gain of the 20 MHz channels evaluated over 9900 measured channels (100 measurements and 99 center frequencies).

Fig. $5 b$ (in the middle) studies the fading properties of the links, i.e. the slope of the CPF curves in Fig. 4. The metric chosen here is the $10 \%$ fade depth $F D(10 \%)$ [15]. The $F D(10 \%)$ metric compares the difference between the median value of $P G$ and the $10 \%$ percentile of $P G$ [15]. $F D(10 \%)$ can be directly read from the empirical CPF plots as shown in Fig 4. Despite its simplicity, $F D(10 \%)$ provides a good engineering insight into the nature of fading. For Rayleigh fading, $\quad F D(10 \%)=8.2 \mathrm{~dB}$ whereas for benign Rician channels $F D(10 \%)<8.2 \mathrm{~dB}$ [15]. For $F D(10 \%)>8.2 \mathrm{~dB}$ the channel is Hyper-Rayleigh, i.e. worse than Rayleigh, this occurs typically in channels with two dominant paths [15].

Fig. $5 \mathrm{~b}$ plots $-F D(10 \%)$. The inversion of the axes means that larger area enclosed by the curve equals better fading properties. This is chosen so that the outward direction has the same positive sense for all spider plots.

Fig. $5 \mathrm{~b}$ is an important addition to Fig. 5a because worse median performance can be compensated by more favorable fading properties. This is documented by Fig. 5c (on the right) which represents $P G(5 \%)$, i.e. the path-gain for which the CPFs in Fig. 4 equals 0.05. Comparison between Fig. 5a and Fig. $5 \mathrm{c}$ reveals that the relative ratio between the area enclosed by the red curve (footwear-centric network) and the areas enclosed by the blue and green curves is larger in Fig. 5c.

This is due to better fading properties and it corresponds with Fig. 5b which reports best fading for the footwear-centric architecture. For the hub on the foot, the fading is Rician for all reported measurements $(F D(10 \%)<8.2 \mathrm{~dB})$, whereas the head-centric network manifests Rayleigh or Hyper-Rayleigh fading for 4 out of 16 cases $(F D(10 \%) \geq 8.2 \mathrm{~dB})$. For the hub on the waist, the situation is the worst with non-Rician fading
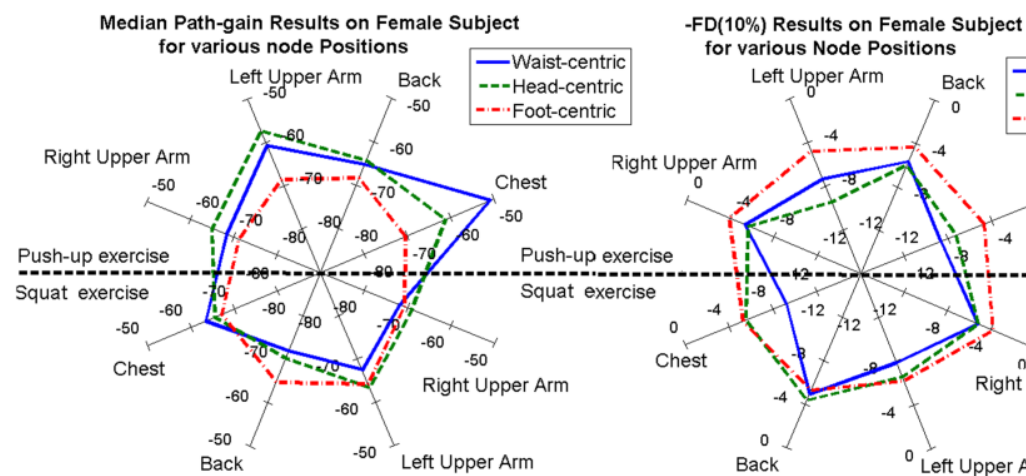

PG(5\%) [dB] Results on Female Subject
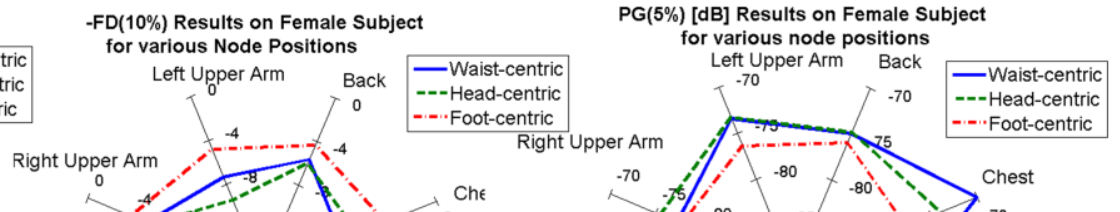

$\tau^{-70}{ }^{-70}$ ----Head-centric
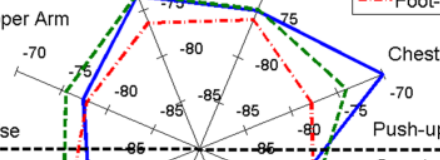

Push-up exercis

Squat exercise
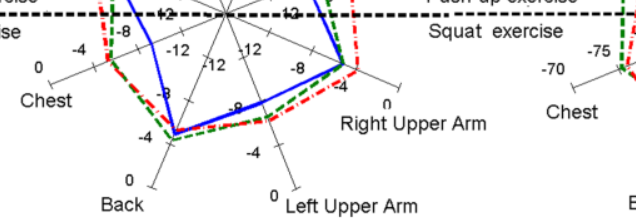

Median Path-gain Results on Male Subject for various node Positions Left Upper Arm Back

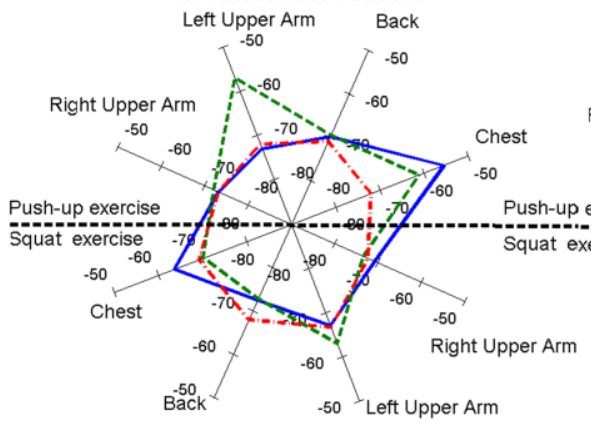

a)

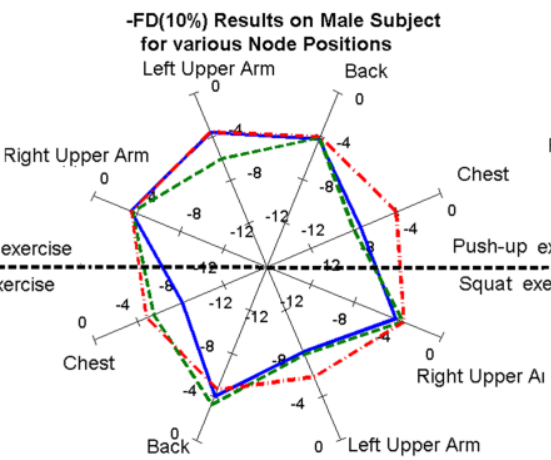

b)

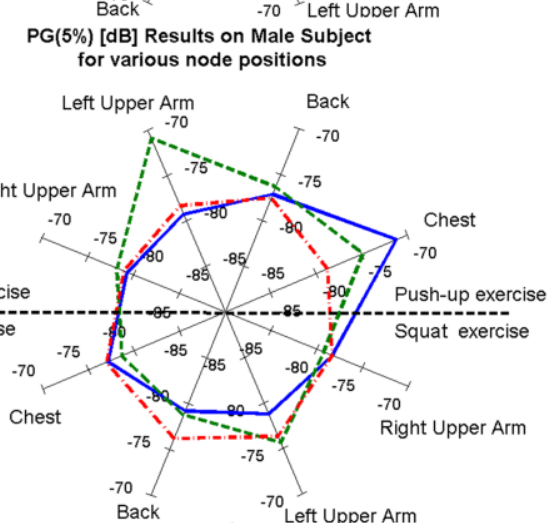

c)

Fig. 5 Spider plots of (a) the median path-gain - summary of the results, (b) $F D(10 \%)$ - description of fading properties, (c) $P G(5 \%)-$ summary of performance guaranteed for $95 \%$ of all measurements 
$(F D(10 \%) \geq 8.2 \mathrm{~dB})$ observed in 7 out of 16 cases.

The superior fading for hubs on the feet and on the temple is present mainly due to the fact that for these locations the use of directional antennas is possible. These antennas focus the energy around the body and limit the impact of multipath, which is not possible for hubs on the hip/torso which require omnidirectional antennas.

Additionally, the case with hub on the foot shows the smallest difference between the male and female measurements. Both subjects differed in size and in the way the exercises were performed. This has impact on the shape of the curves between corresponding plots. The differences are the smallest for the footwear-centric architecture. This suggests that the performance of this network will be affected the least when deployed on different subjects.

In contrast, the footwear centric architecture manifests the worst performance for push-up. This is because the antenna's orientation relative to the ground is and the proximity of the ground does not allow establishment of a wave that efficiently creeps around the body [8].

Overall, the best location to place a hub for fitness scenario is the head, because it offers better fading performance than the waist and in terms of minimum guaranteed path-gain $P G(5 \%)$, it has the best performance in 10 out of 16 cases.

The runner-up is the foot-wear centric network which, thanks to the Rician fading, guarantees a $P G(5 \%)$ path-gain equal or better than the waist-centric system in 11 out 16 cases. However, it guarantees path-gain better than the headcentric network only in 2 out of 16 cases.

\section{CONCLUSION}

This letter compared the propagation properties of WBANs for three locations of the hub on the human body (waist, head, and foot) for a fitness scenario, i.e. for subjects exercising squats and push-ups. The best overall performance is achieved for hub location on the left temple. The worst performance is then provided by the waist-centric system, mainly due to the worst fading properties of such a channel.

These results further confirm previous works [7]-[9], which pointed out that the waist/hip may not be the most suitable location for hubs in future WBANs because other locations allow the use of directive antennas which reduce the impact of fading and improve the overall path-gain characteristics.

To our best knowledge the letter is the first to introduce the concept of spider plots for evaluation of WBANs properties. This enables efficient comparison of different hub locations on the human body and therefore represents a powerful framework for future research searching application specific optimum for hub location. Future work will focus on the expanding the study for off-body networks to explore the impact of the hub location on off-body links.

\section{ACKNOWLEDGMENT}

This work was supported by the Science Foundation Ireland grant 09/IN.1/I2652 and by the Government of Ireland Postdoctoral Fellowship in Engineering, Science and Technology from the Irish Research council.

\section{REFERENCES}

[1] S. Carlaw, "Emerging Bluetooth Verticals," in Bluetooth World 2013, Shanghai, China, 2013,

[2] N.F. Timmons, W.G. Scanlon, W.G., "Analysis of the performance of IEEE 802.15.4 for medical sensor body area networking," in Sensor and Ad Hoc Communications and Networks, Santa Clara, 2004, pp.16-24

[3] A. Alomainy, Y. Hao, C.G. Parini, P.S. Hall, "Comparison between two different antennas for UWB on-body propagation measurements," IEEE Antennas and Wireless Propag. Lett., vol.4, pp.31-34, 2005

[4] S.L. Cotton, W.G. Scanlon, "An experimental investigation into the influence of user state and environment on fading characteristics in wireless body area networks at $2.45 \mathrm{GHz}$," IEEE Trans. Wireless Commun., vol.8, no.1, pp. 6-12, Jan. 2009

[5] A. Fort, J. Ryckaert, C. Desset, P. De Doncker, P. Wambacq, L. Van Biesen, "Ultra-wideband channel model for communication around the human body," IEEE Sel. Areas in Commun., vol.24, no.4, pp.927-933, Apr 2006

[6] Q.H. Abbasi, A. Sani, A. Alomainy, Yang Hao, "On-Body Radio Channel Characterization and System-Level Modeling for Multiband OFDM Ultra-Wideband Body-Centric Wireless Network," IEEE Trans. Microw. Theory and Tech., vol.58, no.12, pp.3485-3492, Dec 2010.

[7] D. Gaetano, V. Sipal, P. McEvoy, M.J. Ammann, C. Brannigan, L. Keating, F. Horgan, "Footwear-centric body area network with directional UWB antenna," Electronics Letters, vol.49, no.14, pp.860861, Jul 42013

[8] D. Gaetano, "Analysis and Design of Footwear Antennas, " $\mathrm{PhD}$. dissertation, Dublin Institute of Technology, Dublin, Ireland, Sep 2014

[9] V. Sipal, D. Gaetano, P. McEvoy, M. J. Ammann, " Bandwidth Scaling of Fading Properties of On-body Wireless Channel in Body Area Networks," in Communications 2014, Bucharest, Romania, 2014

[10] Jie Dong, D. Smith, "Opportunistic relaying in wireless body area networks: Coexistence performance," in IEEE International Conference Communications, pp. 5613 - 5618, Budapest, Hungary, 2013

[11] A. Cihangir, W.G. Whittow, C.J. Panagamuwa, F. Ferrero, G. Jacquemod, F. Gianesello, C. Luxey, "Feasibility Study of 4G Cellular Antennas for Eyewear Communicating Devices," IEEE Antennas and Wireless Propag. Lett., vol.12, no., pp.1704-1707, 2013

[12] G. Leen, D. Heffernan, "Vehicles without wires," Computing \& Control Engineering Journal, vol.12, no.5, pp.205-211, Oct. 2001

[13] R. van Nee, "Wireless LAN -Quo vadis?, " in European Conference on Antennas and Propagation, The Hague, The Netherlands, 2014

[14] T. P. Gareau, R.G. Smith, M. E. Barbercheck, D. A. Mortensen, "Spider Plots: A Tool for Participatory Extension Learning," Journal of Extension, vol. 48, no. 5, Oct 2010

[15] J. Frolik, V. Sipal, D.J. Edwards, "Leveraging Depolarization to Increase the Link Reliability for Wireless Sensors Operating in Hyper-Rayleigh Environments," IEEE Sensors J., vol.14, no.8, pp.2442-2446, Aug 2014 